\title{
Editorial
}

\section{Frames and Overcomplete Representations in Signal Processing, Communications, and Information Theory}

\author{
Radu V. Balan, ${ }^{1}$ Yonina C. Eldar, ${ }^{2}$ and Thomas Strohmer ${ }^{3}$ \\ ${ }^{1}$ Siemens Corporate Research, 755 College Road East, Princeton, NJ 08540, USA \\ ${ }^{2}$ Department of Electrical Engineering, Technion-Israel Institute of Technology, Haifa 32000, Israel \\ ${ }^{3}$ Department of Mathematics, University of California, 1 Shields Avenue, Davis, CA 95616-8633, USA
}

Received 3 September 2005; Accepted 3 September 2005

Copyright () 2006 Radu V. Balan et al. This is an open access article distributed under the Creative Commons Attribution License, which permits unrestricted use, distribution, and reproduction in any medium, provided the original work is properly cited.

\begin{abstract}
Many problems in signal processing, communications, and information theory deal with linear signal expansions. The corresponding basis functions are typically orthogonal (nonredundant) signal sets. It is well known that the use of redundancy in engineering systems improves robustness and numerical stability. Motivated by this observation, redundant linear signal expansions (also known as "frames") have found widespread use in many different engineering disciplines. Recent examples include sampling theory, A/D conversion, oversampled filter banks, pattern classification, multiple description source coding, wavelet-based and framebased denoising, and space-time coding for wireless communications.

This special issue of EURASIP JASP brings together researchers from areas as diverse as harmonic analysis, image processing, and wireless communications by combining invited papers with regular contributions related to these topics.
\end{abstract}

The papers in this issue are broadly classified into four main areas:

(1) frame theory,

(2) sparse representations,

(3) filter banks and sampling,

(4) applications.

Each area is represented by several papers that sometimes span overlapping territories.

The first paper in the category of frame theory, by J. J. Benedetto and J. D. Kolestar, develops methods for constructing Grassmannian frames in 2 and 3 dimensions and reviews many of the prior results on this problem. The existence and properties of chirps over finite groups is the focus of the work of P. G. Casazza and M. C. Fickus. In the next paper, Y. C. Eldar and O. Christensen develop an alternative parametrization of all dual frame sets of a given frame and specialize this description to shift-invariant frames. A. Feuer et al. construct a unified transform to analyze linear timeinvariant systems from the viewpoint of frame theory. The paper by S. D. Howard et al. investigates the finite Heisenberg-Weyl group and its ubiquitous role in radar, communications, and the theory of error-correcting codes. In the final paper in this category, J.-B. Martens surveys the Hermite transform, which can be used for overcomplete representation of signals, treating both theory and applications.

The next two papers focus on sparse representations, a topic of intense current research efforts. M. Elad presents uniqueness results regarding sparse signal decompositions in a probabilistic framework. The paper by A. K. Fletcher et al. addresses the problem of denoising by sparse approximation and develops bounds on the mean-squared approximation error, for both deterministic and random dictionaries.

Filter banks and sampling theory are the topic of the third group of papers. The first paper in this series, by $\mathrm{P}$. T. Boufounos and A. V. Oppenheim, explores the use of projections onto synthesis frame vectors and the issue of frame-vector ordering. The next paper, by B. Dumitrescu et al., presents an efficient algorithm for designing oversampled modulated filter banks. The paper by $\mathrm{H}$. Johansson and P. Löwenborg studies the problem of reconstruction of band-limited signals from uniform samples and introduces a reconstruction method based on time-varying finite-length discrete-time filters. S. Marinkovic and C. Guillemot consider joint source-channel coding via an oversampled filter bank code and apply their method to a wavelet-based image coding system. C. Siclet et al. present a theoretical analysis of oversampled DFT modulated transmultiplexers and analyze associated design criteria. Finally, the paper by S. Weiss et al. proposes an oversampled filter bank design algorithm 
for channels with known noise covariance that minimizes the output noise power subject to a normalization constraint.

We conclude this special issue by a series of papers focusing on applications of frame theory. The paper by R. Bernardini et al. considers an application of frame expansions to multiple description video coding exploiting the error recovery capabilities of frame expansions. M. M. Hartmann et al. introduce the concept of multipulse multicarrier modulation, a wireless communication scheme that has its roots in multiwindow Gabor systems. The next article by F. Jin et al. proposes a new denoising method in which motion estimation and compensation, as well as temporal and spatial filtering, are all done in the wavelet domain. Another interesting application area is psychoacoustic analysis. In this context, the paper by R. B. Reilly proposes a tone-frequency linear representation of acoustic data designed specifically to accommodate the nonlinear phenomenon of beats. The next two papers by K. Skretting and J. H. Husøy and by J. E. Vila-Forcén et al. make use of overcomplete dictionaries to select an optimum representation: the texture classifier in the first paper uses sparse linear representations in a supervised learning fashion, whereas the facial image encoder in the second paper uses the edge process model to achieve higher compression rates. In the final paper of this special issue, Y. Sriraja and T. Karp propose a SPIHT algorithm which incorporates a new interpolation scheme able to partially recover lost data.

\section{ACKNOWLEDGMENTS}

We would like to thank all our colleagues who have contributed to this special issue, including the authors of submitted papers. We also thank the reviewers for their quality work, Dr. Helmut Bölcskei for inviting us to edit this special issue, and finally Dr. Marc Moonen and the Editorial Board; without their support this special issue would not have been possible.

Radu V. Balan

Yonina C. Eldar

Thomas Strohmer
Yonina C. Eldar received the B.S. degree in physics in 1995 and the B.S. degree in electrical engineering in 1996, both from Tel Aviv University (TAU), Tel Aviv, Israel, and the Ph.D. degree in electrical engineering and computer science in 2001 from Massachusetts Institute of Technology (MIT), Cambridge. From January 2002 to July 2002, she was a Postdoctoral Fellow at the Digital Signal Processing Group, MIT.

She is currently an Associate Professor with the Department of Electrical Engineering, Technion-Israel Institute of Technology, Haifa, Israel. She is also a Research Affiliate with the Research Laboratory of Electronics at MIT. Her current research interests are in the general areas of signal processing, statistical signal processing, and quantum information theory. Dr. Eldar was in the program for outstanding students at TAU from 1992 to 1996. In 1998, she held the Rosenblith Fellowship for studies in electrical engineering at MIT, and in 2000, she held an IBM Research Fellowship. She is currently a Horev Fellow of the Leaders in Science and Technology program at the Technion and an Alon Fellow. In 2004, she was awarded the Wolf Foundation Krill Prize for Excellence in Research, and in 2005 the Andre and Bella Meyer Lectureship. She is a Member of the IEEE Signal Processing Theory and Methods Technical Committee and an Associate Editor for the IEEE Transactions on Signal Processing.

Thomas Strohmer got his M.S. and Ph.D. degrees in mathematics in 1991 and 1994, respectively, from the University of Vienna, Austria. He was a Research Assistant at the Department of Mathematics, University of Vienna, from 1991 to 1997 . He spent one year as an Erwin-Schroedinger Fellow at the Department of Statistics at Stanford University and then joined the Department of Mathematics at the University of California

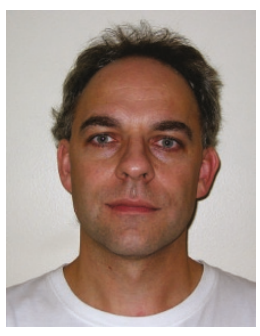
in Davis in 1998, where he is now a Full Professor. His general research interests are in harmonic analysis, numerical analysis, digital signal processing, and wireless communications. He is the coeditor of two books and on the editorial board of several journals. He also serves as a consultant to the telecommunications industry.

Radu V. Balan holds a B.S. degree in EE control theory (1992) from Polytechnic Institute of Bucharest, a B.S. degree in theoretical physics (1994) from University of Bucharest (Romania), and a Ph.D. degree in applied mathematics (1998) from Princeton University, NJ. After one year of postdoctoral research at the IBM T. J. Watson and the IMA University of Minnessota (19981999), Radu Balan joined Siemens Corpo-

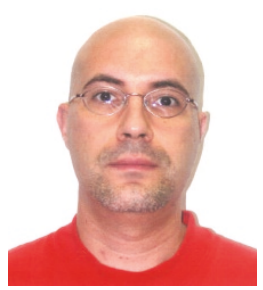

rate Research in Princeton, NJ, where he currently is a member of the technical staff and an Adjunct Lecturer at Princeton University. His interests include applied harmonic analysis (frames and Gabor analysis), signal processing (audio and speech, microphone array, blind source separation, sensor fusion), and classification theory (SVM, kernel methods). 